\title{
Spatial dynamics of invasive Carduus thistles
}

\author{
E. Jongejans $^{\text {a }}$, M.R. Allen ${ }^{\text {b }}$, A.E. Leib ${ }^{\text {c }}$, K.M. Marchetto ${ }^{\text {d }}$, N.M. Pedatella ${ }^{\text {e }}$, J. Peterson-Smith ${ }^{\text {c }}$, \\ E.S.J. Rauschert ${ }^{\mathrm{f}}$, D.C. Ruggiero ${ }^{\mathrm{c}}$, L.A. Russo ${ }^{\mathrm{c}}$, L.E. Ruth ${ }^{\mathrm{g}}$, Z. Sezen ${ }^{\mathrm{h}}$, O. Skarpaas ${ }^{\mathrm{i}}$, \\ B.J. Teller ${ }^{c}$, L.A. Warg ${ }^{j}$, S. Yang ${ }^{c}$, R. Zhang ${ }^{c}$, and K. Shea $^{c}$ \\ ${ }^{a}$ Institute for Water and Wetland Research, Radboud University Nijmegen, Nijmegen, the Netherlands; \\ ${ }^{b}$ Department of Animal Ecology, University of Illinois at Urbana-Champaign, Urbana, IL, USA; \\ ${ }^{c}$ Department of Biology, Pennsylvania State University, University Park, PA, USA; ${ }^{d}$ Department of Ecology \\ and Evolutionary Biology, Cornell University, Ithaca, NY, USA; ${ }^{e}$ National Center for Atmospheric \\ Research, Boulder, CO, USA; ${ }^{f}$ Department of Crop and Soil Sciences, Pennsylvania State University, USA; \\ ${ }^{g}$ College of Agricultural Sciences, Pennsylvania State University, USA; ${ }^{h}$ Department of Entomology, \\ University of Minnesota, St. Paul, MN, USA; ${ }^{i}$ Norwegian Institute for Nature Research, Oslo, Norway; \\ ${ }^{j}$ Medical Scientist Training Program, University of Colorado, USA. Email: E.Jongejans@science.ru.nl
}

Abstract: Managing invasive species is inherently a spatial problem, even though the application of management is local. For efficient management of invasive species, we must therefore understand their spatial population dynamics. Once key demographic and dispersal rates are studied in detail, spatial population models can be constructed and utilized to evaluate the impact of various management options.

We illustrate this approach for the invasive thistles Carduus acanthoides and Carduus nutans in North America, where these Eurasian plants arrived in the $19^{\text {th }}$ century. The invasion routes through North America were reconstructed from a literature review: within 150 years both species have reached most US states and Canadian provinces. At the continental scale, these high invasion rates are most likely caused by human transport. However, within states the distributions are patchy. At this regional/local scale, wind dispersal of the plumed seeds is also important. Therefore, at the scale of neighboring farms, management can be informed by mechanistic population models that incorporate both wind and human dispersal.

One of the foundations of a spatial population model is the local dynamics of a species. By revisiting known populations three years later, we show that initial size of $C$. nutans populations has a strong impact on the persistence and growth of these populations. More than fifty percent of small populations went extinct in those three years, whereas larger populations had higher persistence rates. This can be attributed to demographic stochasticity, but experimental results suggest that this can also be partly explained by reduced pollinator services in small thistle populations. Local population dynamics can be studied in more detail by following the fate of individuals and by relating the performance of individuals to their size. The resulting size-structured population models have indeed proven to be useful for studying thistle populations.

Another key component of spatial population models is dispersal. We have been studying all key aspects of the dispersal process in these Carduus thistles: seed release, movement, and impact upon arrival. The seed release phase has been mainly studied in wind tunnel experiments which show that it is possible to predict the timing of seed release based on flowering phenology and weather data. Furthermore, field experiments on seed dispersal have shown that the resulting probability density kernel of dispersal distances can be well fitted by a semi-mechanistic WALD model. This allows us to simulate realistic dispersal kernels based on wind speed, turbulence, release and vegetation height, and seed terminal velocity.

Now these demography and dispersal components of the spatial population dynamics need to be integrated. We have started doing so with spatially-implicit matrix and integral projection models. We expect that the field of invasion ecology will move towards more process-oriented, transparent, analytical models with increasing biological realism. Alternatively the same demography and dispersal functions can be used in spatially-explicit population models that trade tractability for the flexibility to also include the effects of heterogeneous landscape configurations and species interactions on both local population dynamics and seed dispersal.

Keywords: Dispersal, Pollination, Population dynamics, Seed release, Spatial integral projection model 


\section{INTRODUCTION}

Invasive alien plant species can negatively affect native biodiversity and can have large management costs in both nature reserves and agricultural settings. Understanding how invasive plants spread through the invaded range is therefore pivotal for efficient control management. Spatial population models that include demographic and dispersal processes in detail can be used to identify the key processes that contribute most to population spread rates, and to select management options that are most likely to be effective.

In this paper we give an overview of what is known about the demography, dispersal and invasion history of Carduus thistles in various landscapes. We present new data on the persistence of populations and conclude by outlining how these pieces of information can be used in analytical spatial population models and in spatially-explicit simulations.

\subsection{Species}

The invasive Eurasian thistles Carduus acanthoides and C. nutans are monocarpic perennials that live for one or more years as a rosette, then bolt, flower and die. Their plumed seeds are wind-dispersed. Germination mostly occurs in the fall and vernalization is required for flowering. These invasive thistles typically occur in agricultural fields, pastures, lawns, roadsides or disturbed areas in general, and have large environmental and economic impacts (Dunn 1976; Skinner et al 2000). Biological control attempts have had variable success in North America, Australia and New Zealand (Shea and Kelly 1998; Groenteman et al. 2011).

\section{INVASION HISTORY}

\subsection{Literature search}

The presence of $C$. nutans was first recorded in the United States in 1853 near Harrisburg, Pennsylvania, while it was first recorded in Canada in New Brunswick in 1871 (Desrochers 1988). An extensive literature search was conducted in order to track the North American spread of $C$. nutans and $C$. acanthoides since their introduction (Leib 2009). Approximately 200 floras published between 1850 and 2008 were consulted to identify relevant information about the presence (or absence) of the thistles. Other books, herbarium records, journal articles, databases and websites were also consulted. Of the various sources, a majority contained no information concerning the thistles. Others contained information on when the thistles were first noted at the state level, county level, or at even higher spatial resolutions. From these records maps of North America with first occurrence of the thistles by state or province were constructed (Fig. 1). The maps showed that, after the initial appearances on the east coast, the thistle invasions were sporadic. Several sources suggested thistles spread along roads and railroad, in roadsides and neglected or overgrown fields. Correlation analysis, however, showed that thistle appearance was more strongly related to milk production (and hence pasture cover) than to railroad construction among states (Leib 2009).
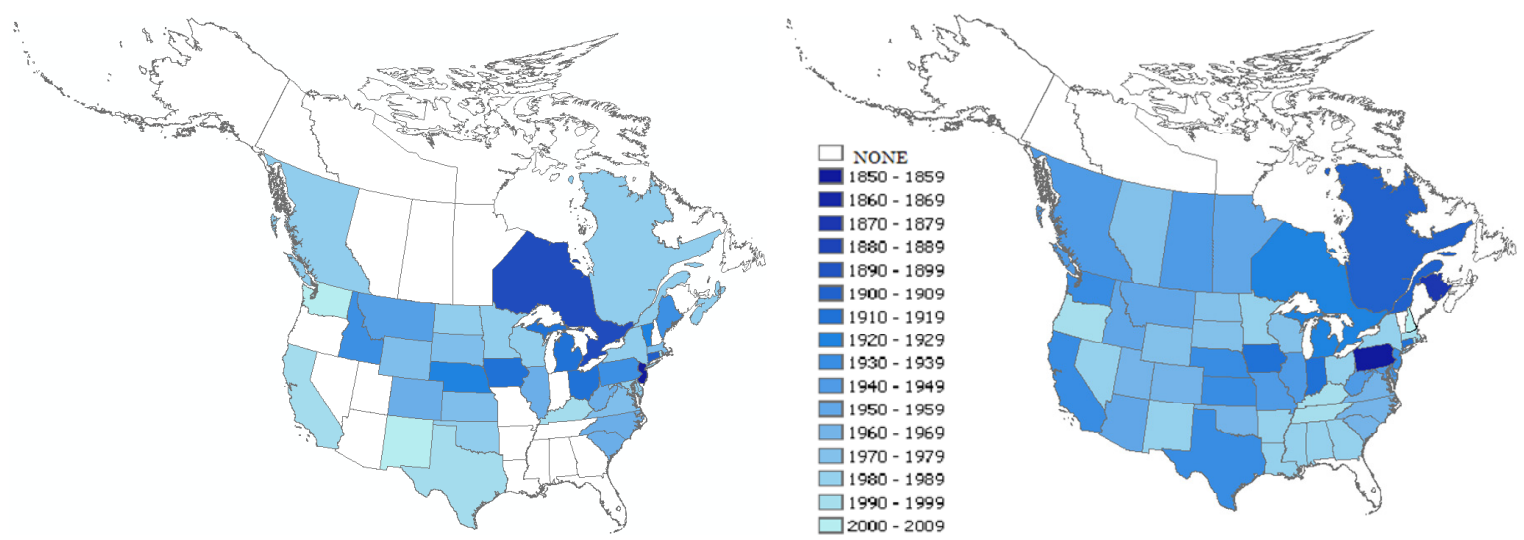

Figure 1. Map of North America showing first records of C. acanthoides (left) and C. nutans (right) by U.S. state and Canadian province (maps from Leib 2009).

\subsection{Preliminary genetic analysis}

In a preliminary genetic study (Warg 2008), DNA from samples originating in the Netherlands, United Kingdom, and France were compared with DNA from samples collected in Pennsylvania and Oregon in order to identify the origin of the invading populations. Single nucleotide polymorphisms and 
insertions/deletions were identified through sequencing of chloroplast spacer regions. According to maximum parsimony analysis, Oregon samples were closely related to Pennsylvania samples, suggesting that plants residing on the East coast spawned the West coast invasion. Alternately, the Oregon thistles could have resulted from a second invasion from the same Eurasian parent population that produced the Pennsylvania thistles. The maximum parsimony tree indicated that this parent population was likely located in France. More samples and sequencing of additional loci are necessary to confirm this hypothesis.

\subsection{Regional distribution patterns}

While both $C$. nutans and $C$. acanthoides have spread across the North American continent over the last 150 years, population distributions are much patchier on a regional scale. For instance, in a $5000 \mathrm{~km}^{2}$ study conducted in central Pennsylvania, sizes of individual patches ranged from one individual to thousands, often with large distances between them. Additionally, there was little overlap in the two species' distributions across this range (Allen and Shea 2006), even though each species' range extended in all directions. When examining fields of natural co-occurrence, there was strong aggregation both within and between species, most likely as a result of clumping in favorable habitat (Rauschert et al 2011). The emerging picture is that thistles continue to spread at the regional level. Understanding this 'infilling' is crucial for management.

\section{LOCAL POPULATION DYNAMICS}

Demographic studies have been performed in several natural $C$. nutans populations: the growth and fate of individual plants have been recorded in 3 populations in the south of France (Département Aveyron; Sheppard et al. 1989), 2 in Southeast Australia (Shea et al. 2005), 2 on the North Island of New Zealand (Shea and Kelly 1998), and 2 in Kansas, USA (Lee and Hamrick 1983). The individual-level data from these field studies provide estimates of vital rates like survival, growth, flowering and seed production. Life cycle diagrams bring together these vital rates to form a matrix model of population size dynamics. Comparative analyses with transition matrix population models show that the populations studied in the invaded ranges grew more rapidly than those in the native range (France) because of higher seed production potential and lower seed losses to specialist insects destroying developing seeds (Jongejans et al. 2008). Some populations in the invaded ranges also had increased individual growth and flowering rates. Additional demographic data comes from common garden studies in which plants were grown without (initial) competition, mimicking overgrazed or highly disturbed sites. Under such conditions $C$. nutans was capable of very fast growth and producing tens of thousands of seeds (Jongejans et al. 2008). Another field experiment with C. acanthoides showed that the number of seeds produced by the first colonizing thistle was very important for the development of a population in subsequent years (Jongejans et al. 2007b).

\subsection{Revisiting populations to assess the change in population size over 3 years}

Although demographic field studies are very informative of the processes governing population dynamics, they are also timeconsuming and the number of populations that can be studied is therefore limited. To study the fate of a larger number of populations we adopted a simpler approach: in 2005 we revisited 162 sites where Allen and Shea (2006) encountered C. nutans populations in 2002. In both the initial and subsequent census population size was categorized as either 1-10, $11-50$ or $50+$ individuals $(81,54$ and 27 populations, respectively). After 3 years the fate and size of the populations varied strongly depending on their initial sizes (Fig. 2). More than half of the small (1-10 flowering plants)
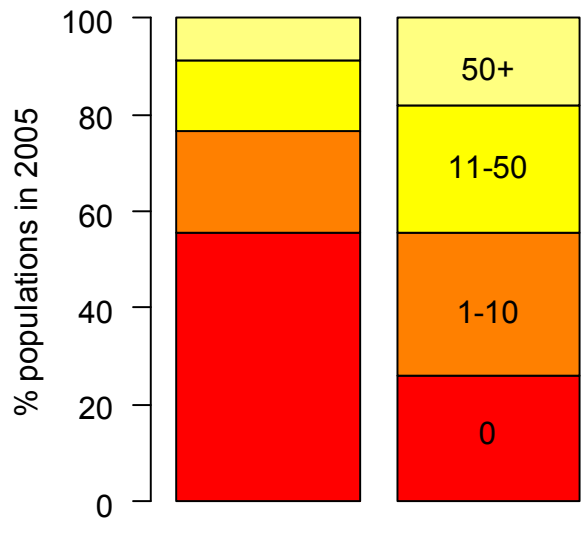

$$
1-10
$$$$
11-50
$$

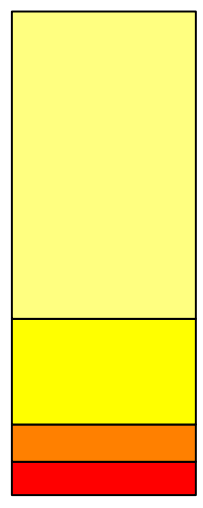

$50+$

$$
\text { population sizes (\# flowering plants) in } 2002
$$

Figure 2. Changes in the size of Pennsylvanian $C$. nutans populations over a 3-year period populations were extinct (i.e. no flowering plants were found, though we could not exclude a remaining seed bank or small rosettes). On the other hand, large (50+) populations only had a 5-10\% extinction rate and had a more than $60 \%$ chance of maintaining a large number of individuals. While many extinctions had probably occurred through succession and deliberate eradication, others disappeared as a result of land use change. 


\section{SPATIALLY VARIABLE DEMOGRAPHIC PROCESSES}

Several aspects of the life history of thistles involve spatial processes: specialist herbivores have to find plants that are preparing to flower, pollinators have to travel between individual flowering plants and between populations, surviving seeds disperse away from their mother, and seeds arrive in variable microsite conditions, which co-determine the probability of seedling establishment and plant growth. We have studied each of these spatially variable aspects, as discussed in the following subsections.

\subsection{Pollination}

Both C. acanthoides and C. nutans are pollinated by insects such as bees and butterflies (Giurfa and Núñez 1992). A study in Pennsylvania showed that the pollinator community consisted primarily of Bombus species ( $73 \%$ of visits). At lower rates, Syrphid flies (15\%), honey bees (6\%), moths and butterflies $(2 \%)$, ants (2\%), and solitary bee species (2\%) were also observed on thistle flower heads (L.A. Russo, unpublished data). Although both plant species are similarly self-compatible (Warwick 1987), pollination between flowers on the same and different plants is accomplished through their insect pollinators (Jessep 1990). Pollination of both species appears to be limited by population size. Small, experimental populations of potted plants had lower fitness (here: seed set), either because of inbreeding depression, pollinator limitation or both (Yang et al. 2011). Larger populations of flowering plants tend to increase pollinator visitation (e.g., Ågren 1996; Sih and Baltus 1987), though isolated C. acanthoides and C. nutans individuals received sufficient pollinator services to set approximately $50 \%$ of their seeds (Yang et al. 2011). Thus, reductions in seed production due to pollinator limitation can be large for small and isolated populations of the thistles. Consideration of pollinator behavior may be a critical part of modeling the spatial spread of these two Carduus species.

\subsection{Seed release}

Non-random seed release can have a strong impact on the distances traveled by seeds (Schippers and Jongejans 2005). This is because the dispersal vector (horizontal and vertical air flow) varies strongly within the dispersal season (Skarpaas et al. 2011), and because the dispersal unit (plumed seed) is affected by humidity (falling faster to the ground when wet). Field observations on potted C. acanthoides plants showed that seeds release mostly between 10 a.m. and 4 p.m., times when temperatures and wind speeds are higher and humidity is lower (N.M. Pedatella, unpublished data). Increased wind speed and turbulence had a positive effect on seed release in wind tunnel trials (Skarpaas et al. 2006; Jongejans et al. 2007a). Characteristic interactions between flower heads and airflow, revealed by particle image velocimetry, demonstrate fine-scale mechanisms for these effects (Marchetto et al. 2010b). Prolonged humid conditions, however, may hinder seed release (Jongejans et al. 2007a). Characteristics of the flower heads themselves, such as head diameter and the number of seeds previously released, also had significant effects on seed release. Seed release was also affected by environmental conditions (e.g. drought) experienced by the mother plant (B.J. Teller, unpublished data).

\subsection{Seed dispersal}

The movement of seeds after release from the mother plant has been mostly studied with respect to dispersal by wind. Field studies with seed traps at distances up to $120 \mathrm{~m}$ from thistle patches showed mean dispersal distances of $2 \mathrm{~m}$, but also further dispersal up to $96 \mathrm{~m}$ (Skarpaas and Shea 2007). The dispersal kernels from that research were well fitted by semi-mechanistic Wald Analytical Long-distance Dispersal models that are partly based on estimates of seed release height, vegetation height, falling speed of seeds, and wind speed parameters. Dispersal distances were also measured by tracking single seeds that were released by hand. These tracking studies resulted in similar mean distances but less variation around the mode (Skarpaas et al. 2011a). The wider and flatter dispersal kernels from the trapping studies can be attributed to the fact that the seed trap studies sampled a much longer time period (weeks) and natural seed release dynamics, whereas the tracking studies were limited to several hours at a time.

\subsection{Seedling establishment}

In studies of seedling emergence and establishment of $C$. nutans and $C$. acanthoides, emergence and subsequent survival increased with microsite size, and were strongly improved with a tilling disturbance (Peterson-Smith and Shea 2010; Ruggiero and Shea 2011). The effects of microsite competition can persist throughout the life-cycle, although they may impact stages differently (Rauschert and Shea, in review). Press (tilling and weeding of interspecific competitors) rather than pulse (tilling alone) disturbances did increase the emergence of $C$. nutans, but did not significantly alter rosette size, suggesting that seedling establishment 
is the life history stage most sensitive to interspecific competition. These results suggest that interspecific competition for a resource other than photosynthetically active radiation (PAR) may be the limiting factor in emergence; without disturbance, the emergence of $C$. nutans in forested microsites was over $35 \%$ but less than $1 \%$ in the pasture, despite higher PAR in the undisturbed pasture microsite (Peterson-Smith and Shea 2010). Although emergence was relatively high in the forest, no seedlings survived the drop of leaves in fall.

\subsection{Seed bank}

Many weed species have a documented ability to produce seed banks, where dispersed seeds remain dormant and do not germinate immediately (Christoffoleti and Caetano 1998, Forget et al 2005). A recent experiment tested the longevity of $C$. acanthoides and $C$. nutans seed survival by burying 90 packets of 50 seeds at a depth of $5 \mathrm{~cm}$ underground. A subset of packets were exhumed 9 times over 2 years to test the remaining seeds for germination potential and for viability using a tetrazolium test. The number of live seeds decreased with time, but viable seeds still remained for both species after 2 years (Ruth 2009).

\subsection{Specialist herbivores}

Several specialist herbivores of Carduus thistles have been released for biological control in their invaded range (Groenteman et al. 2011). The dynamics of a biological control agent relative to the dynamics of its host is an important determinant of the success of this type of management. In addition to high attack rates on the host, a successful biological control agent needs to exhibit positive spatial density dependence in order to affect local population growth. We studied the response of a biological control agent Rhinocyllus conicus, a flower head weevil, to the local spatial distribution of $C$. nutans. We found that $R$. conicus was able to find host plants based on patch characteristics but oviposition (attack) rates were dependent on plant characteristics (Sezen 2007). Despite an apparent lack of effective biological control at the local scale, results of a pseudo-spatial metapopulation model of $C$. nutans and $R$. conicus showed that the presence of $R$. conicus in the landscape reduced the proportion of the landscape occupied by C. nutans significantly (Sezen 2007).

\section{INTEGRATION AND DISCUSSION}

We use spatial models to assess how important different demography and dispersal processes are for local, regional and continental scale spread. Spatial models that include these demographic and dispersal mechanisms in sufficient detail can be used to generate insights for management. In this section we discuss spatially-implicit model analyses we have performed for homogeneous landscapes and plans for spatiallyexplicit simulations for studying spread through heterogeneous landscapes.

\subsection{Spatially-implicit models of population spread}

An elegant method for integrating demography and dispersal was introduced by Neubert and Caswell (2000): integrodifference equations for invasion speed. These spatially-implicit Neubert-Caswell models (NCMs) combine the matrix models discussed in section 3 with dispersal kernels, and allow for an analytical comparison of the contributions of all demographic and dispersal parameters (Lewis et al. 2006). Using the French, Australian, New Zealand, Kansas and Pennsylvania matrix models (section 3), and supplementing these with wind data and seed falling speeds from those states, Jongejans et al. (2008) showed that the modeled spread rates of $C$. nutans populations in the invaded ranges were higher than in the native range (after the effect of insect herbivores was removed). In some invasive populations increased growth, flowering and/or seed production were the primary contributors to the higher spread rates, while in other invasive populations, dispersal parameters like taller plants and reduced falling speeds made major contributions.

These NCMs for $C$. nutans have been used to study how different management strategies affect local population growth and spread in different invaded ranges. Shea et al. (2010) showed that the effects of herbicides, grazing and biocontrol agents depend on which life history parameters are affected and how sensitive population growth and spread are to changes in those parameters. As a result, three biological control agents (insect species) reduced thistle spread rates differently between Australia and New Zealand.

Another example of the applicability of NCMs is provided by Zhang et al. (2011) who found that simulated climate warming increased the number of flower heads per plant as well as seedling emergence, and to a lesser degree plant survival and plant height. These experimental findings were integrated with an NCM based on demographic data from a previous experiment in the same field. Model analysis showed that in contrast to the order of the empirical effect sizes, the change in the dispersal parameter plant height caused by far the biggest boost of invasion speed. This suggests that interannual weather variation can impact spread patterns and that higher spread rates can be expected in warmer regions. It will therefore be interesting to 
study interannual variation in this system with recently developed stochastic NCMs (Caswell et al. 2011). Furthermore, continuous state variables allow for a more detailed study of size-dependent effects, as has been shown for C. nutans in spatial integral projection models (Jongejans et al. 2011).

\subsection{Spatial-explicit models of population dynamics in heterogeneous landscapes}

Although the analytical approaches discussed in the previous section have the advantage of being very tractable and allowing easy comparison between populations and across studies, landscapes are rarely homogeneous as assumed in NCMs. To study effects on the spread of thistles in heterogeneous landscapes we plan to use spatially-explicit model simulations. These models can be parameterized with precisely the same demography and dispersal routines developed for the NCMs, but require additional information such as the landscape configuration of different habitat types and habitat-specific demographic and dispersal rates. As evidenced by section 4, such data are available for many aspects of the life history of $C$. acanthoides and C. nutans. Furthermore, population size feeds back to landscape parameters. For example, Marchetto et al. (2010a) demonstrated that thistle patch size influences wind speeds and thereby the dispersal of seeds. Spatially-explicit simulations will also allow for studying the importance landscape-dependent, wind-driven seed release and dispersal functions (Damschen et al. 2008). Spatially-explicit individual-based models also present the opportunity to study the interaction between the spatial population dynamics of the thistles, their pollinators and biological control agents.

\section{ACKNOWLEDGMENTS}

We are grateful for support by many undergraduate students in the Shea lab. The Netherlands Organization for Scientific Research (NWO-veni grant 863.08.006 to EJ), and the National Science Foundation of the USA (NSF grants DEB-0315860 to KS \& OS and DEB-0614065 to KS \& EJ) provided funding.

\section{REFERENCES}

Ågren, J. (1996). Population size, pollinator limitation, and seed set in the self-incompatible herb Lythrum salicaria. Ecology, 77, 1779-1790.

Allen, M.R. and Shea, K. (2006). Spatial segregation of congeneric invaders in central Pennsylvania, USA. Biological Invasions, 8, 509-521.

Caswell, H., Neubert, M.G. and Hunter, C.M. (2011). Demography and dispersal: invasion speeds and sensitivity analysis in periodic and stochastic environments. Theoretical Ecology, in press.

Christoffoleti, P.J. and Caetano, R.S.X. (1998). Soil seed banks. Scientia Agricola 55.

Damschen, E.I., Brudvig, L.A., Haddad, N.M., Levey, D.J., Orrock, J.L. and Tewksbury, J.J. (2008). The movement ecology and dynamics of plant communities in fragmented landscapes. Proceedings of the National Academy of Sciences of the United States of America, 105, 19078-19083.

Desrochers, A.M., Bain, J.F. and Warwick, S.I. (1988). The biology of Canadian weeds 89. Carduus nutans L. and Carduus acanthoides L., Canadian Journal of Plant Science, 68, 1053-1068.

Dunn, P.H. (1976). Distribution of Carduus nutans, C. acanthoides, C. pycnocephalus, and C. crispus, in the United States. Weed Science, 24, 518-524.

Forget, P.M., Lambert, J.E., Hulme, P.E. and Vander Wall, S.B. (2005). Seed Fate: Predation, Dispersal, and Seedling Establishment. CABI publishing, Oxfordshire, UK.

Giurfa, M. and Nuñez, J.A. (1992). Foraging by honeybees on Carduus acanthoides: pattern and efficiency. Ecological Entomology, 17, 326-330.

Groenteman, R., Kelly, D., Fowler, S.V. and Bourdôt, G.W. (2011). Abundance, phenology and impact of biocontrol agents on nodding thistle (Carduus nutans) in Canterbury 35 years into a biocontrol programme. New Zealand Journal of Agricultural Research, 54, 1-13.

Jessep, C.T. (1990). Aspects of the biology of nodding thistle (Carduus nutans L) in Canterbury, New Zealand. New Zealand Journal of Agricultural Research, 33, 173-183.

Jongejans, E., Pedatella, N.M., Shea, K., Skarpaas, O. and Auhl, R. (2007a). Seed release by invasive thistles: the impact of plant and environmental factors. Proceedings of the Royal Society B, 274, 24572464.

Jongejans, E., Shea, K., Skarpaas, O., Kelly, D., Sheppard, A.W. and Woodburn, T.L. (2008). Dispersal and demography contributions to population spread of Carduus nutans in its native and invaded ranges. Journal of Ecology, 96, 687-697.

Jongejans, E., Shea, K., Skarpaas, O., Kelly, D. and Ellner, S.P. (2011). Importance of individual and environmental variation for invasive species spread: a spatial integral projection model. Ecology, 92, 86-97. 
Jongejans, E., Skarpaas, O., Tipping, P.W. and Shea, K. (2007b). Establishment and spread of founding populations of an invasive thistle: the role of competition and seed limitation. Biological Invasions, 9 , 317-325.

Lee, J.M. and Hamrick, J.L. (1983). Demography of two natural populations of musk thistle (Carduus nutans). Journal of Ecology, 71, 923-936.

Leib, A.E. (2009). Analysis of the distribution of Carduus nutans and Carduus acanthoides in North America since 1853. Honor's BSc thesis, Pennsylvania State University.

Lewis, M.A., Neubert, M.G., Caswell, H., Clark, J.S. and Shea, K. (2006). A guide to calculating discretetime invasion rates from data. Conceptual Ecology and Invasion Biology: Reciprocal Approaches to Nature. (eds M.W. Cadotte, S.M. McMahon \& T. Fukami), p. 169-192. Springer.

Marchetto, K.M., Jongejans, E., Shea, K. and Isard, S.A. (2010a). Plant spatial arrangement affects projected invasion speeds of two invasive thistles. Oikos, 119, 1462-1468.

Marchetto, K.M., Williams, M.B., Jongejans, E., Auhl, R. and Shea, K. (2010b). Applications of particle image velocimetry for seed release studies. Ecology, 91, 2485-2492.

Neubert, M.G. and Caswell, H. (2000). Demography and dispersal: calculation and sensitivity analysis of invasion speed for structured populations. Ecology, 81, 1613-1628.

Peterson-Smith, J. and Shea, K. (2010). Seedling emergence and early survival of Carduus spp. in three habitats with press and pulse disturbances. Journal of the Torrey Botanical Society, 137, 287-296.

Rauschert, E.S.J., Shea, K. and Bjørnstad, O.N. (2011). Coexistence patterns of two invasive thistle species, Carduus nutans and C. acanthoides, at three spatial scales. Biological Invasions, in press.

Ruggiero, D.C. and Shea, K. (2011). Effects of interspecific competition on early life history of the invasive thistles Carduus nutans and C. acanthoides. Northeastern Naturalist, 18, 197-206.

Ruth, L.E. (2009). Carbon/nitrogen allocation and seed bank retention of two invasive Carduus thistles. Honor's BSc thesis, Pennsylvania State University.

Schippers, P. and Jongejans, E. (2005). Release thresholds strongly determine the range of seed dispersal by wind. Ecological Modelling, 185, 93-103.

Sezen, Z. (2007). Interactions of the invasive thistle Carduus nutans and its biocontrol agent Rhinocyllus conicus in heterogeneous environments. $\mathrm{PhD}$ thesis, Pennsylvania State University.

Shea, K., Jongejans, E., Skarpaas, O., Kelly, D. and Sheppard, A.W. (2010). Optimal management strategies to control local population growth or population spread may not be the same. Ecological Applications, 20, 1148-1161.

Shea, K. and Kelly, D. (1998). Estimating biocontrol agent impact with matrix models: Carduus nutans in New Zealand. Ecological Applications, 8, 824-832.

Shea, K., Kelly, D., Sheppard, A.W. and Woodburn, T.L. (2005). Context-dependent bological control of an invasive thistle. Ecology, 86, 3174-3181.

Sheppard, A.W., Cullen, J.M., Aeschlimann, J.-P., Sagliocco, J.-L. and Vitou, J. (1989). The importance of insect herbivores relative to other limiting factors on weed population dynamics: a case study of Carduus nutans. Proceedings of the VII International Symposium on Biological Control of Weeds, 611 March 1988, Rome, Italy (ed. E.S. Delfosse), pp. 211-219. Ministero dell'Agricoltura e delle Foreste Rome/CSIRO, Melbourne, Australia.

Sih, A. and Baltus, M.S. (1987). Patch size, pollinator behavior, and pollinator limitation in catnip. Ecology, 68, 1679-1690.

Skarpaas, O., Auhl, R. and Shea, K. (2006). Environmental variability and the initiation of dispersal: turbulence strongly increases seed release. Proceedings of the Royal Society B, 273, 751-756.

Skarpaas, O. and Shea, K. (2007). Dispersal patterns, dispersal mechanisms, and invasion wave speeds for invasive thistles. American Naturalist, 170, 421-430.

Skarpaas, O., Shea, K., and Jongejans, E. (2011). Watch your time step: trapping and tracking dispersal in autocorrelated environments. Methods in Ecology and Evolution, 2, 407-415.

Skinner, K., Smith, L. and Rice, P. (2000). Using noxious weed lists to prioritize targets for developing weed management strategies. Weed Science, 48, 640-644.

Warg, L.A. (2008). Tracking the spread of the invasive thistle Carduus nutans using single nucleotide polymorphisms. Honor's BSc thesis, Pennsylvania State University.

Warwick, S.I. (1987). Estimates of outcrossing rates in pure populations and hybrid swarms of Carduus nutans and Carduus acanthoides. American Journal of Botany, 74, 763.

Yang, S., Ferrari, M.J., and Shea, K. (2011). Pollinator behavior mediates negative interactions between two congeneric invasive plant species. American Naturalist, 177, 110-118.

Zhang, R., Jongejans, E. and Shea, K. (2011). Warming increases the spread of an invasive thistle. PLoS ONE, 6, e21725. 